\title{
Algunos aspectos biológicos del falso cordón de fraile Hyptis capitata Jacq en el Departamento del Meta
}

\author{
Some biological aspects of the friar false cord Hyptis capitata Jacq into Meta \\ Department \\ Melón Quevedo Carlos Alberto; Ríos Ríos Ariel Rene ${ }^{1}$; Bastidas Harold ${ }^{2}$ y \\ Rincón Ricardo ${ }^{1}$ \\ ${ }^{1}$ Ingeniero Agrónomo y ${ }^{2}$ Ingeniero Agrónomo, MSc \\ hbastidas@unillanos.edu.co
}

Recibido 15 de Mayo 2013, Aceptado 15 de Julio 2013

\section{RESUMEN}

Este trabajo se realizó a nivel de invernadero y campo en la Universidad de los Llanos sede Barcelona, municipio de Villavicencio departamento del Meta, con una latitud norte de $4^{\circ} 3^{\prime}$ y $63^{\circ} 38^{\prime}$ longitud oeste, el clima corresponde a bosque húmedo tropical con una humedad relativa del $75 \%$ y una precipitación promedio anual 2500 milímetros, con distribución bimodal. El objetivo general de este trabajo es conocer algunas características biológicas, fisiológicas y respuesta a herbicidas de la planta Hyptis capitata Jacq en el departamento del Meta. Se realizaron pruebas de escarificación, latencia de semillas, viabilidad de semilla, profundidad de emergencia, morfología y biología. También se realizaron pruebas de análisis bromatológico y mineralógico. Además, se utilizaron pruebas de efectos de herbicidas en pre-emergencia y post-emergencia. Adicionalmente se realizaron pruebas de esta planta como hospedero de enemigos naturales, en un diseño de bloques completos al azar con 4 repeticiones, se utilizó como cultivo acompañante y se midió la presencia de enemigos naturales y la incidencia de parasitoides sobre algunas plagas de arroz. La germinación de la especie es mayor en la superficie ó hasta los dos centímetros de profundidad. La capacidad de persistencia de esta especie no radica en su latencia, ó en su porcentaje de germinación ya que son muy bajas; sino, en la capacidad de producir una alta 
cantidad de semillas (más de 3000 semillas por planta). Por otro lado, dentro de la alta cantidad de especies de insectos que llegan a las plantas de Hyptis, las que se presentan en mayor cantidad son de los órdenes Hemíptera, Coleóptera, Himenóptera y de la clase Arácnida, los cuales poseen un alto número de beneficios entre sus representantes como Reduviideae, Calcideae, Pompilideae, entre otras. Así mismo, si se emplean herramientas de tipo químico para el control de Hyptis capitata Jaqc, dentro de los pre-emergentes el de mejor desempeño es el oxadiazon y butaclor. Los mejores herbicidas post-emergentes para el control de falso cordón de fraile son Azimsulfuron + Metsulfuronmetil, Byspiribae Sodio y Metsulfuronmetil. Por último, la población de Trichogramma spp, Telenomus y moscas de la Familia Tachinidae fue mayor en la distribución de $H$. capitata en los bordes y en cruz.

Palabras clave: Maleza, herbicida, germinación, persistencia, cultivo.

\begin{abstract}
This work was performed at the level of greenhouse and field at the Universidad de los Llanos, Barcelona seat in Villavicencio municipality, Meta department, with a north latitude of $4^{\circ} 3$ 'and $63^{\circ} 38^{\prime}$ west longitude, the climate is a tropical rainforest relative humidity of $75 \%$ and an average annual rainfall $2500 \mathrm{~mm}$, with bimodal distribution. The overall objective of this study was to determine some biological characteristics, physiological and herbicide response of the plant Hyptis capitata Jacq into Meta department. Prick tests were conducted, seed dormancy, seed viability, emergency depth, morphology and biology. Tests were also conducted mineralogical and chemical composition analysis. Furthermore, tests were used effects of herbicides in pre-emergence and post-emergence. Further tests were conducted this plant as a host of natural enemies, in a design of randomized complete block with 4 replicates, was used as a companion crop and measured the presence of natural enemies and parasitoids on the incidence of some pests of rice. Germination of the species at the surface is greater or up to two inches deep. Persistence ability of this species lies in its latency, or in the germination
\end{abstract}


percentage is very low and that, but in the ability to produce a high amount of seeds (more than 3000 seeds per plant). Furthermore, within the high amount of insect species arriving Hyptis plants, which are present in greater quantity in the Hemiptera, Coleoptera, Hymenoptera orders and Arachnid class, which have a high number of benefits among their representatives Reduviideae, Calcideae, Pompilideae, among others. Also, if using tools of chemical to control Hyptis capitata Jaqc within the pre-emerging the top performer is the oxadiazon and butachlor. The best post-emergent herbicide for control of friar false cord are Azimsulfuron + Metsulfuronmetil, Byspiribae Sodium and Metsulfuronmetil. Finally, the population of Trichogramma spp, and flies Telenomus, Tachinid family was greater in the distribution of $H$. capitata on the edges and cross.

Keywords: Weed, herbicide, germination, persistence, cultivation.

\section{INTRODUCCIÓN}

Las malas hierbas son uno de los principales factores que causan disminución en los rendimientos y aumento en los costos de producción, al igual que la incidencia de plagas y enfermedades en el sector agrícola (Gómez, 1993). Así mismo, el desconocimiento de aspectos biológicos de las malezas y el inadecuado manejo de las mismas, ha ocasionado un aumento considerable en las poblaciones; al igual que la diseminación de especies nocivas a lo largo y ancho del país, favoreciendo el desarrollo de ciertas especies que comienzan a ser consideradas como problema importante en el cultivo del arroz, como es el caso de falso cordón de fraile Hyptis capitata Jacq. La falta de conocimiento de las características biológicas de las especies como Hyptis capitata y la importancia que ha adquirido como planta nociva en el cultivo del arroz, o sus posibles efectos benéficos alelopáticos como lo hace en otros cultivos (palma africana); hace que se generen dudas sobre su manejo, además que desconocer sus características biológicas y fisiológicas repercute en no lograr tomar decisiones adecuadas a la hora de su control. 
Según Bastidas y Ordóñez, (2001) el falso botoncillo, $H$. capitata es la maleza de mayor presencia en cohortes de poblaciones de malezas en cultivos de arroz en la región del Ariari con rangos que oscilan entre 40 y 200 plantas por metro cuadrado. Teniendo como base el hecho de la poca información existente sobre biología de esta planta, se hace necesario obtenerla para así tener bases concisas que permitan desarrollar métodos de manejo, uso y control de Hyptis capitata Jacq. Se reporta un uso potencial como planta utilizada en corredores biológicos como atrayente y refugio de enemigos naturales de Lepidópteros, plagas especialmente en palma africana, y este trabajo puede ofrecer algunos resultados para ser incorporados en planes de agricultura biológica o de sostenibilidad (Calvache et al., 2000).

El objetivo general fue conocer algunas características biológicas, fisiológicas y respuesta a herbicidas de la planta Hyptis capitata Jacq en el Departamento del Meta. Dentro de los objetivos generales se determinó el periodo de latencia en semillas de Hyptis capitata; se determinó el efecto de la profundidad de emergencia sobre la germinación de semillas; se cuantificó el número de semillas promedio por planta; se conoció el mejor método de escarificación de semilla de $H$. capitata; se analizó la eficiencia de herbicidas en pre-emergencia y postemergencia bajo condiciones controladas sobre $H$. capitata Jacq. Se conoció la composición mineralógica y bromatológica de la especie en estudio para inferir los posibles nutrientes de extracción en un lote; y se determinó la presencia de insectos en la planta de Hyptis capitata, al igual que la frecuencia de visita de los insectos a nivel de campo.

Según Cronquist, (1992) del total de las especies, alrededor de 255.000, 30.000 son dañinas en algún grado para el hombre, de éstas unas doscientas cincuenta especies se consideran malezas de importancia económica ya que afectan la capacidad de producción de las plantas cultivadas, interfiriendo con su desarrollo normal por la competencia y/o por los efectos alelopáticos que provocan (Gómez y Rivera, 1987). 
Según Takhtajan, (1997) la clasificación taxonómica de Hyptis capitata es la siguiente: Reino: Plantae; División: Magnoliophyta; Clase: Magnoliopsida; Subclase: Lamiidae; Superorden: Laminae; Orden: Lamiales; Familia: Lamiaceae; Género: Hyptis; Especie: Hyptis capitata Jacq.

El falso cordón de fraile es una planta dicotiledónea, anual, herbácea de 0.30 a 1.0 metros de altura, raíz pivotante, el tallo grueso cuadrangular, erecto, ramificado con pilosidad esparcida (Marzocca, 1985). Las hojas opuestas con pecíolo corto a largo, ovadas a un poco lanceoladas y de pubescentes a glabras; de 3 a $6 \mathrm{~cm}$. de largo por 2 a $6 \mathrm{~cm}$. de ancho, bordes aserrados (Cárdenas et al., 1972). Según Gómez y Rivera, (1987) la inflorescencia es axilar, en grupos compactos, opuestos, densos, semejantes a cabezuelas y con brácteas ovado-lanceoladas y pubescentes, las flores inconspicuas, numerosas, pequeñas, blancas o verdeblancuzcas, a veces lilas, cada una con un cáliz más o menos simétrico, rectopubescentes por fuera y por dentro, el fruto está conformado por 3 a 4 nuececillas ovadas a oblongas de color café claro a marrón; se propagan por semilla. Crece en zonas con altitudes entre 0 y 1800 m.s.n.m. y temperatura superior a 17.5 grados centígrados (Salamanca et al., 2001)

Trabajos realizados en Ecuador con 130 especies para análisis fitoquímico y farmacológico, arrojaron resultados prometedores para cuatro especies entre las cuales se encuentra Hyptis capitata (Ghia, 1993). Así mismo, en los últimos años, CENIPALMA ha realizado la evaluación de catorce especies de plantas atrayentes de insectos benéficos, entre los que sobresalen dos especies del género Hyptis; (Hyptis mutabilis e Hyptis capitata) (Calvache et al., 2000). Estas plantas crecen en suelos desnudos y en sitios soleados. Los principales grupos de insectos visitantes son avispas Braconidae, Eucharitidae, Pteromalidae y Vespidae (Mexzon y Chinchilla, 1999).

\section{METODOLOGÍA}

Este trabajo se realizó a nivel de invernadero y campo en la Universidad de los Llanos sede Barcelona, municipio de Villavicencio departamento del Meta, con una 
latitud Norte de $4^{\circ} 3^{\prime}$ y $63^{\circ} 38^{\prime}$ longitud Oeste, el clima corresponde a bosque húmedo tropical, una humedad relativa del $75 \%$ y una precipitación promedio anual 2500 milímetros, con distribución bimodal.

\section{Ensayo 1. Escarificación}

Se evaluaron diez tratamientos de rompimiento de latencia, para cada tratamiento se realizaron diez repeticiones, la unidad experimental fue de cincuenta semillas sembradas en vasos desechables de $10 \mathrm{~cm}$ de profundidad, en suelo esterilizado en autoclave por tres horas con 15 atmósferas de presión, los resultados se midieron en porcentaje de germinación quince días después de la siembra con un diseño completamente al azar, se realizó un análisis de varianza y comparación de medias siguiendo la metodología utilizada por Jara y Turriago, (1997).

Tabla 1. Escarificación para semillas de H. capitata

\begin{tabular}{cl}
\hline Tratamiento & \multicolumn{1}{c}{ Descripción } \\
\hline 1 & Agua a $\mathrm{T}^{\circ}$ ambiente por 24 horas \\
2 & Agua a $\mathrm{T}^{\circ}$ ambiente por 48 horas \\
3 & Agua a $\mathrm{T}^{\circ}$ ambiente por 12 horas \\
4 & Estufa $\mathrm{a} 65^{\circ}$ por 48 horas. \\
5 & Estufa a $65^{\circ}$ por 72 horas. \\
6 & $\mathrm{HCl}$ al $0.01 \%$ por 5 minutos. \\
7 & $\mathrm{H} 2 \mathrm{SO} 4$ al $0.01 \%$ por 5 minutos. \\
8 & Agua a 65 OC por 3 minutos \\
9 & Agua a $100^{\circ} \mathrm{C}$ por 3 minutos \\
10 & Testigo \\
\hline
\end{tabular}

\section{Ensayo 2. Profundidad de emergencia}

El ensayo se realizó en materas de $20 \mathrm{~cm}$ de alto, cada tratamiento con tres repeticiones con una unidad experimental de 50 semillas escarificadas de $H$. capitata sembradas a profundidades de 0,2, 5, 8 y $10 \mathrm{~cm}$, el diseño utilizado fue completamente al azar. Se evaluaron la emergencia cada tres días hasta completar 30 días después de la siembra, siguiendo la metodología utilizada por Jara y Turriago 1997; Bastidas 1993). 


\section{Ensayo 3. Viabilidad en semillas de Hyptis capitata}

Luego de la recolección en campo de una alta cantidad de semillas de $H$. capitata; se sembraron semanalmente cincuenta (50) semillas con cinco (5) repeticiones y se contaron la cantidad de semillas emergidas. Este procedimiento se realizó hasta obtener porcentajes de germinación muy bajos similares a cero, obteniendo así una curva de viabilidad.

\section{Ensayo 4. Morfología y crecimiento de Hyptis capitata}

Altura: Se tomo como referencia el tallo principal desde la base hasta el último nudo de la última hoja emergida. A partir de 8 días después de la emergencia hasta floración. Materia seca: Se midió la producción de materia seca promedio al tomar tallo, hojas y raíz de quince plantas cada ocho días desde la germinación hasta inicio de la floración; se pesaron húmedas y luego se secaron en la estufa a una temperatura de $65^{\circ} \mathrm{C}$ hasta que se obtuvo peso constante. La información se suministra como materia seca en gramos por planta y en porcentaje de materia seca. Número de semillas por planta: Se tomaron cincuenta plantas representativas del campo y se contó el número de inflorescencias, a su vez que se contó la cantidad de semilla de cincuenta inflorescencias con el fin de obtener promedios y de allí el número de semillas por planta. Número de hojas: De cada planta se midió semanalmente el número de hojas fotosintéticamente activas; este ensayo constó de cinco repeticiones, se evaluaron los promedios de los resultados. Análisis bromatológico: Se realizó en el laboratorio de nutrición animal de la Universidad De Los Llanos Orientales, siguiendo la metodología de AOAC, (2006). Análisis mineralógico: El análisis mineralógico de $H$. capitata se realizó según la metodología de Jiménez y Ballesteros, (1989).

\section{Ensayo 5. Valoración e incidencia de $\boldsymbol{H}$. capitata en poblaciones de insectos}

Valoración visual de insectos benéficos sobre Hyptis capitata: En este ensayo se realizaron cuatro muestreos de 10 pases dobles de jama en sitios 
donde la población de $H$. capitata era representativa; estos jameos se realizaron dos de 7 a 10 a.m. y dos de 3 a 6 p.m. por un periodo de ocho días. Las muestras obtenidas fueron sometidas a identificación a nivel de familia.

Incidencia de $\boldsymbol{H}$. capitata en las poblaciones de insectos benéficos en el cultivo de arroz: El experimento se realizó con un diseño de bloques completos al azar con cuatro repeticiones, el tamaño de la parcela fue de $4 \mathrm{~m} \times 5 \mathrm{~m}\left(20 \mathrm{~m}^{2}\right)$. Se sembró la variedad de arroz Fedearroz 50 con una densidad de $200 \mathrm{Kg} / \mathrm{Ha}$ de semilla al voleo. La fertilización se realizó de acuerdo al análisis de suelos. No se aplicaron productos químicos insecticidas en ninguna etapa del cultivo. Los tratamientos fueron los siguientes: 1. Arroz sin cultivo acompañante, 2. Arroz con Hyptis capitata en los cuatro bordes, 3. Arroz con $H$. capitata en dos bordes y Arroz con Hyptis en cruz en el interior de la parcela. Las plantas de Hyptis capitata se sembraron 12 días antes que el arroz, con una densidad de 200 plantas $/ \mathrm{m}^{2}$. Los parámetros evaluados fueron: Población de insectos en el cultivo del arroz, 10 pases dobles de jama en cada parcela con una frecuencia de 10 días empezando a partir de los 8 días después de la siembra (Metodología según Pantoja et al., 1999).

\section{Ensayo 6. Efecto de herbicidas sobre H. capitata}

\section{Pruebas de preemergencia}

Luego de sembradas las semillas, se aplicaron los productos comerciales con una bomba tipo $Z$ a una presión de tres bares y doscientos litros de agua/ha (Tabla 2). Las pruebas se realizaron con 50 semillas por matera con tres repeticiones por tratamiento, diseño completamente al azar; se evaluó emergencia en porcentaje, tiempo de emergencia y descripción visual del efecto de los herbicidas sobre las plantas de H. capitata.

\section{Pruebas de post emergencia}

El ensayo se realizó con plantas entre dos y cuatro hojas las cuales se sembraron en materas de $20 \mathrm{~cm}$. de profundidad, cada tratamiento con tres repeticiones 
(Tabla 3); se evaluó sintomatología y porcentaje de eficiencia, las observaciones se realizaron cada cinco días después de la aplicación por un periodo de 20 días, los herbicidas se aplicaron con la bomba tipo z, el diseño fue completamente al azar, se efectuó un análisis de varianza y comparación de medias.

Tabla 2. Herbicidas pre-emergentes utilizados en H. Capitata.

\begin{tabular}{cc}
\hline Ingrediente Activo & Dosis Comercial/ha (L) \\
\hline Pretilaclor & 2,5 \\
Butaclor & 4,0 \\
Oxadiazon & 2,2 \\
Oxyfluorfen & 1,2 \\
Testigo & \\
\hline
\end{tabular}

Tabla 3. Herbicidas post emergentes utilizados en $H$. Capitata.

\begin{tabular}{cc}
\hline Ingrediente Activo & Dosis Comercial/ha \\
\hline $2,4 \mathrm{D}$ & $0.4 \mathrm{~L}$ \\
2.4 D + Picloran & $0.4 \mathrm{~L}$ \\
Metsulfuronmetil & $0,01 \mathrm{~kg}$ \\
Metsulfuronmetil & $0,015 \mathrm{~kg}$ \\
Bentazona & $2,5 \mathrm{~L}$ \\
Loxinil octanoato & $0,3 \mathrm{~L}$ \\
Propanil + Trycorpir & $4,0 \mathrm{~L}$ \\
Propanil & $4,0 \mathrm{~L}$ \\
Propanil + Butaclor & $4,0 \mathrm{~L}$ \\
Byspiribae Sodio & $0,5 \mathrm{~L}$ \\
Testigo & \\
\hline
\end{tabular}

\section{RESULTADOS Y DISCUSIÓN}

\section{Ensayo 1. Escarificación}

Hiptys capitata Jacq posee un porcentaje de emergencia bajo. Se realizó esta prueba con el fin de lograr unos mejores niveles de germinación y disminuir el tiempo de emergencia. Son pocos los tratamientos que representan una diferencia con respecto al testigo, solo el procedimiento de estufa a $65^{\circ} \mathrm{C}$ por 72 horas muestra una diferencia marcada; donde se logró obtener un porcentaje de 
emergencia del $34.2 \%$, seguido por el tratamiento de inmersión de la semilla en agua a $65^{\circ} \mathrm{C}$ por tres minutos con un porcentaje de emergencia de $21.6 \%$ (Tabla $4)$.

Tabla 4. Resultados de tratamientos para prueba de escarificación

\begin{tabular}{lc}
\hline \multicolumn{1}{c}{ Tratamientos } & $\begin{array}{c}\text { Porcentaje de } \\
\text { Germinación }\end{array}$ \\
\hline Agua temperatura ambiente por 48 horas & $5,4^{\mathrm{a}}$ \\
Agua temperatura ambiente por 24 horas & $4,8^{\mathrm{a}}$ \\
Agua temperatura ambiente por 12 horas & $4,0^{\mathrm{a}}$ \\
Estufa $65^{\circ} \mathrm{C}$ por 72 horas & $34,2^{\mathrm{c}}$ \\
Estufa $65^{\circ} \mathrm{C}$ por 48 horas & $16,6^{\mathrm{ab}}$ \\
Ácido sulfúrico $0.01 \%$ por 5 minutos & $7,0^{\mathrm{a}}$ \\
Ácido clorhídrico $0.01 \%$ por 5 minutos & $14,0^{\mathrm{ab}}$ \\
Agua a $100^{\circ} \mathrm{C}$ por 3 minutos & $8,6^{\mathrm{a}}$ \\
Agua a $65^{\circ} \mathrm{C}$ por 3 minutos & $21,6^{\mathrm{bc}}$ \\
Testigo & $5,6^{\mathrm{a}}$ \\
\hline
\end{tabular}

$\mathrm{CV}=112.1 \%{ }^{* *}$ Diferencia altamente significativa $1 \%$.

NOTA: Diferencia con igual letra indica no poseer diferencia estadísticamente significativa.

\section{Ensayo 2. Profundidad de emergencia}

El comportamiento de la especie en estudio, muestra una diferencia de germinación a las diferentes profundidades de siembra; si se analiza el desarrollo de la semilla a cero centímetros de profundidad, se puede apreciar una germinación lenta los primeros 12 días, luego se presenta un aumento en el porcentaje de germinación en un rango entre los 15 y 24 días después de la siembra, para llegar finalmente a su mayor valor entre los 24 y 27 días (Gráfica 1). Si se comparan los resultados de la siembra a cero centímetros con los de las profundidades a dos, cinco y ocho centímetros; se encontró que, estos últimos tratamientos tuvieron un porcentaje de germinación menor, e incluso el inicio y finalización de la misma fue en un corto tiempo; entre los 15 y 18 para dos y cinco centímetros, y entre los 21 y 24 para los ocho centímetros de profundidad. Si se observa detenidamente las curvas, tienden a presentar dos ligeras mesetas, a los nueve días y a los dieciocho días después de la siembra; ello indica que tal como lo reporta la literatura, esta planta posee la facultad de germinar en cohortes 
dándole una posibilidad de evadir aplicaciones de algunos herbicidas, y sugiriendo la necesidad de realizar un análisis de banco de semillas en lotes productivos que posean esta especie.

COMPORTAMIENTO DE GERMINACION DE H. capitata A DIFERENTES PROFUNDIDADES

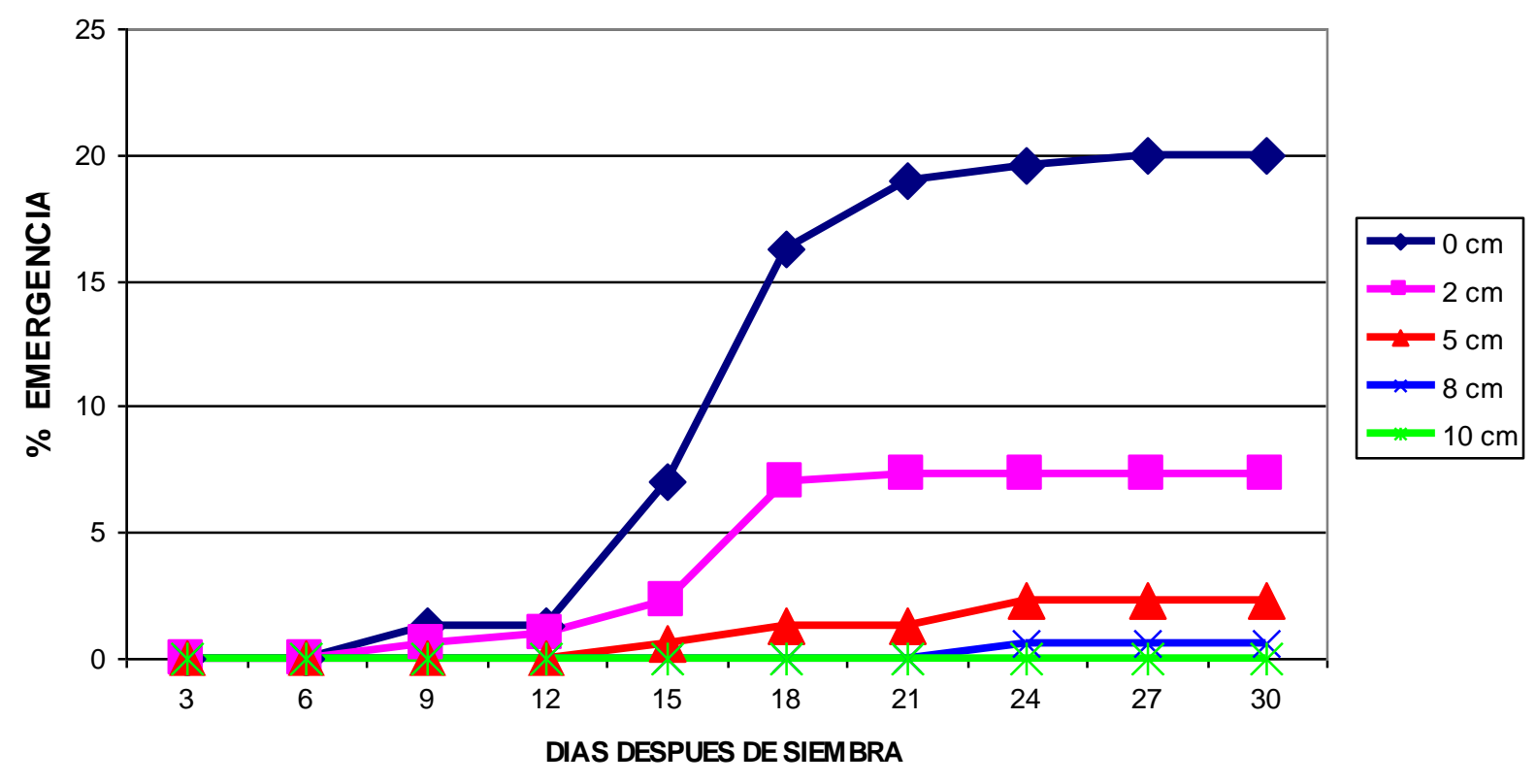

Gráfica 1. Comportamiento de germinación a diferentes profundidades

\section{Ensayo 3. Viabilidad en semillas de Hyptis capitata}

En la Gráfica 2 se observa que la germinación disminuye a medida que transcurre el tiempo, inicia con un porcentaje del 13.6 y a pesar de los picos posee una marcada tendencia de disminuir a cero. Para que esto ocurra transcurren dieciséis semanas, de allí en adelante los ensayos no evidenciaron emergencia de esta especie. Al marcar sobre la gráfica la línea de tendencia, se nota la progresividad de disminuir a cero; además, el ajuste ó mejor la distancia entre puntos hacia la línea de tendencia genera un valor de $R^{2}=0.9259$, indicando una leve variación entre los datos obtenidos; es decir, existen datos alejados de la tendencia (semana 13 por ejemplo), pero en general la información no se alteró estadísticamente. 


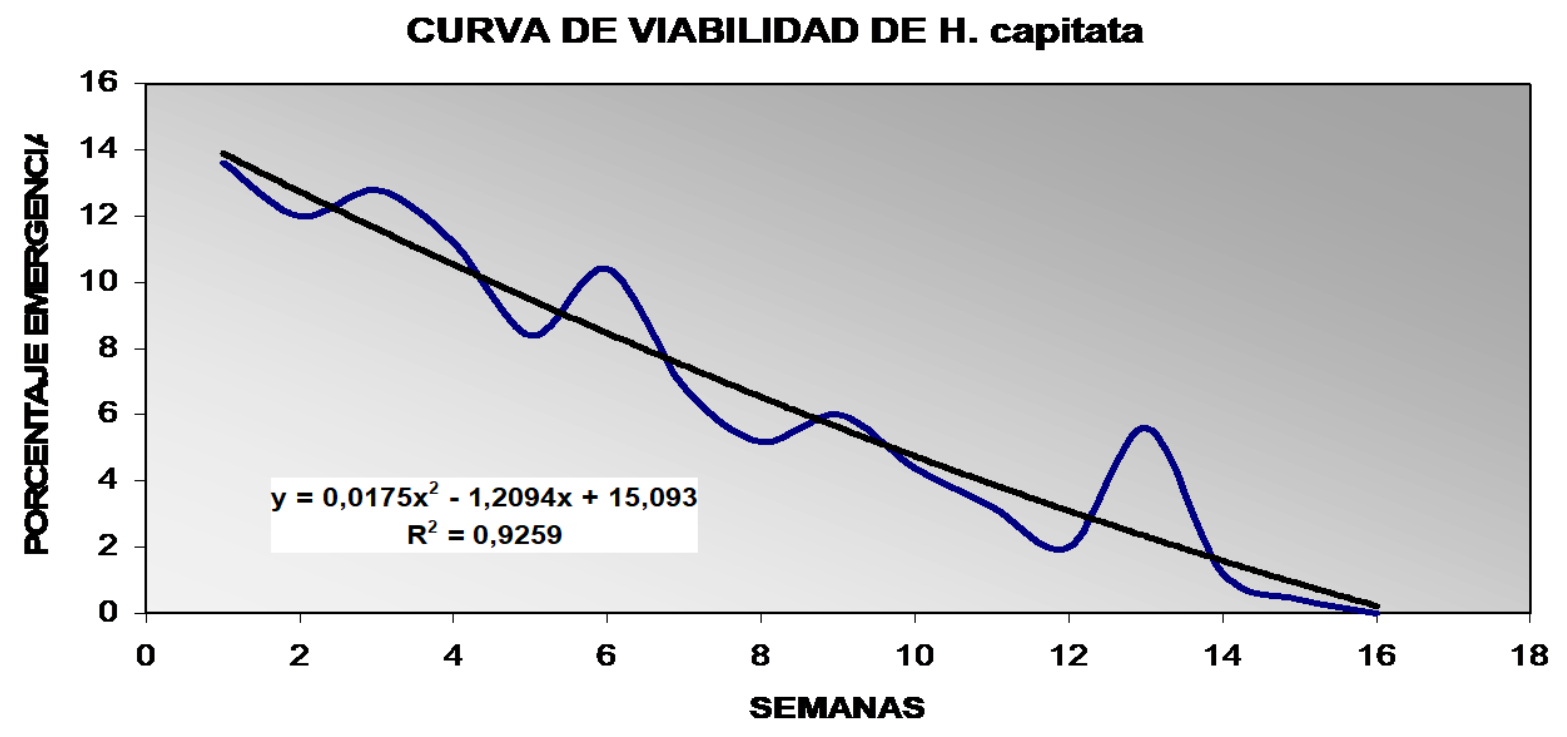

Gráfica 2. Viabilidad de Hyptis capitata a través del tiempo

\section{Ensayo 4. Morfología y crecimiento}

\section{Análisis de materia Seca}

Para la especie Hyptis capitata, se encontró en la primera semana de crecimiento un porcentaje de materia seca del 12.66; el cuál va aumentando progresivamente hasta llegar a un contenido de biomasa de $31.11 \%$ a las ocho semanas donde inicia su etapa reproductiva (Gráfica 3). Este comportamiento refleja que en promedio alcanza una humedad de hasta $70 \%$, lo cual representa un alto grado de lignificación, adaptación en suelos y climas de la región, permitiéndole una capacidad de competencia frente a especies cultivables. Al realizar el trazado de la línea de tendencia se logra inferir una alta similitud entre los datos registrados y graficados $\left(R^{2}=0.964\right)$ indicando un ajuste mínimo hacia la tendencia, la cuál es graficable por la ecuación y $=12.11 x^{04484}$.

H. capitata presentó la primera semana después de la emergencia 5.2 hojas/planta, la segunda semana 9.6 hojas/planta, la tercera semana 12.4 hojas/planta, la quinta semana 20 hojas/planta, la sexta semana 23.6 hojas/planta, la séptima semana 26.4 y la octava semana 30.8 hojas/planta; produce 14 inflorescencias/planta, 229 semillas por inflorescencia y 3196.4 semillas. 


\section{ACUMULACION MATERIA SECA}

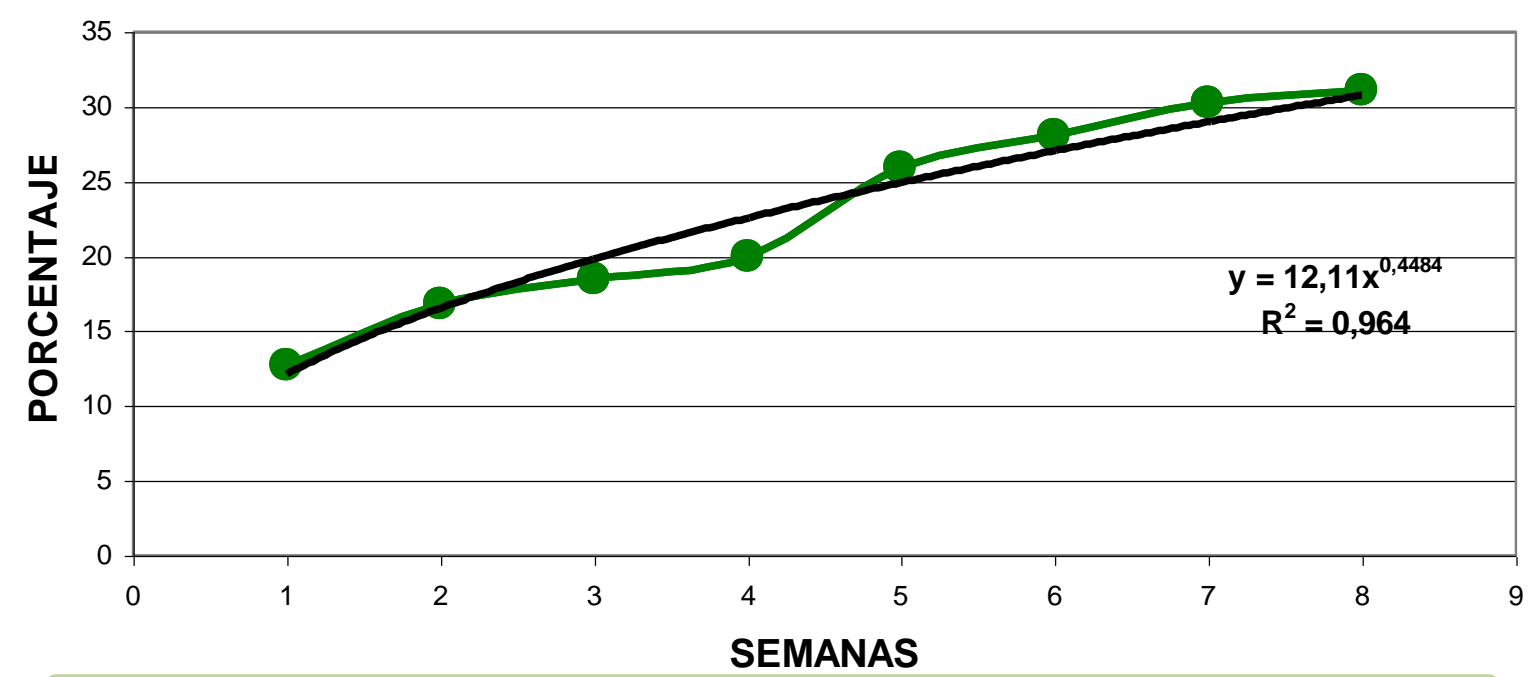

Gráfica 3. Acumulación de materia seca en porcentaje a través del tiempo

\section{Análisis bromatológico}

En términos generales la especie estudiada no es una fuente de alimento para animales rumiantes y ganados, debido a su baja energía digestible y metabólica; además, su contenido de proteína y fibra no es muy representativo. Su contenido de carbohidratos solubles (ENN) es alto (48.05\%) (Tabla 5); esto indica que esta planta posee la capacidad de producir y acumular néctares, lo que la hace atractiva a especies de la clase Insecta, bien sea para alimentarse directamente de ella o de otros insectos (bajo controladores y arácnidos).

\section{Análisis mineralógico}

Al inicio de la floración se estima que las especies poseen acumulada una cantidad representativa de nutrientes extraídos de fuentes como el material orgánico, minerales del suelo y agua. En este punto fenológico de las plantas, los nutrimentos se emplean para floración y muy poco para crecimiento; por tanto, es cuando posee la mayor cantidad de nutrientes acumulados. En este punto se procedió a la toma de muestras de hojas para realizar por medio del laboratorio un 
análisis foliar que permitió estimar su capacidad de absorción de elementos minerales del suelo (Tabla 6).

Tabla 5. Resultados del análisis del estudio bromatológico de $\boldsymbol{H}$. capitata.

\begin{tabular}{lc}
\hline \multicolumn{1}{c}{ Evaluación } & Dato obtenido \\
\hline Cenizas & $9.2 \%$ \\
Grasa & $4.55 \%$ \\
Proteína & $12.02 \%$ \\
Fibra cruda & $21.63 \%$ \\
Extracto no nitrogenado (ENN) & $48.05 \%$ \\
Nutrientes digestibles totales (NDT) & $66.56 \mathrm{Kcal}$. \\
Energía digestible (ED) & $2.93 \mathrm{Kcal}$. \\
Energía metabólica (EM) & $2.41 \mathrm{Kcal}$. \\
Energía bruta (EB) & $3.68 \mathrm{Kcal}$. \\
\hline
\end{tabular}

Tabla 6. Resultado análisis foliar de Hyptis capitata.

\begin{tabular}{|c|c|c|c|c|}
\hline \multicolumn{5}{|c|}{ Micronutrientes en porcentaje (\%) } \\
\hline $\mathrm{Ca}$ & Mg & & $\mathbf{K}$ & $\mathbf{P}$ \\
\hline \multicolumn{5}{|c|}{ Micronutrientes en partes por millón (ppm) } \\
\hline B & $\mathrm{Cu}$ & $\mathrm{Fe}$ & Mn & Zn \\
\hline 16.0 & 12.5 & 37.5 & 100.0 & 25.0 \\
\hline
\end{tabular}

\section{Ensayo 5. Valoración e incidencia en poblaciones de insectos}

Valoración visual de insectos benéficos en $\boldsymbol{H}$. capitata. En un lote representativo de Hyptis capitata, es común observar una gran cantidad de insectos sobre esta especie, básicamente polinizadores, pero también controladores naturales de plagas, por ejemplo el Coleomegila maculata. Se pudo llegar a determinar que los insectos de los órdenes Hemíptera, Coleóptera, Hymenoptera y la clase Arácnida, son de mayor presencia en lotes de Hyptis capitata Jaqc, con una representatividad de 15.3, 7.39, 3.92 y 5 insectos colectados en promedio por muestreo (10 pases dobles de jama) respectivamente Tabla 7). 
Tabla 7. Relación de insectos visitantes de H. capitata.

\begin{tabular}{cc}
\hline $\begin{array}{c}\text { Orden } \\
\text { taxonómico }\end{array}$ & Promedio total insectos colectados \\
\hline Coleóptera & 7.39 \\
Hemíptera & 15.30 \\
Hymenoptera & 3.92 \\
Homóptera & 0.65 \\
Diptera & 1.25 \\
Orthoptera & 0.35 \\
Lepidoptera & 0.40 \\
Odonata & 0.075 \\
Neuroptera & 0.075 \\
Clase Arácnida & 5.00 \\
\hline
\end{tabular}

Incidencia de Hyptis capitata en poblaciones de insectos benéficos en el cultivo de arroz. La población de Aphelinidos y de avispas de la Familia Pompilidae fue similar estadísticamente en la distribución de Hyptis capitata utilizada con rango entre 20 y 22 adultos/día (Tabla 8 ) y presentando diferencias estadísticas con el testigo sin cultivo acompañante (Gráfica 4).

Tabla 8. Relación de insectos benéficos vs distribución de $H$. capitata.

\begin{tabular}{lccccc}
\hline \multicolumn{1}{c}{ Tratamiento } & Testigo & 2 Bordes & 4 Bordes & En cruz & CV \\
\hline Aphelinidos & $8^{\mathrm{a}}$ & $20^{\mathrm{b}}$ & $22^{\mathrm{b}}$ & $20^{\mathrm{b}}$ & 4.5 \\
Trichogramma & $5^{\mathrm{a}}$ & $32^{\mathrm{b}}$ & $40^{\mathrm{c}}$ & $38^{\mathrm{c}}$ & 8.2 \\
Telenomus & $3^{\mathrm{a}}$ & $30^{\mathrm{b}}$ & $38^{\mathrm{c}}$ & $36^{\mathrm{c}}$ & 5.3 \\
Tachinide & $5^{\mathrm{a}}$ & $10^{\mathrm{b}}$ & $22^{\mathrm{c}}$ & $25^{\mathrm{c}}$ & 15.9 \\
Pompilide & $3^{\mathrm{a}}$ & $12^{\mathrm{b}}$ & $12^{\mathrm{b}}$ & $12^{\mathrm{b}}$ & 23.5 \\
Vespide & $5.2^{\mathrm{a}}$ & $10^{\mathrm{b}}$ & $10^{\mathrm{b}}$ & $13^{\mathrm{c}}$ & 14.1 \\
Anthocoride & $3^{\mathrm{a}}$ & $9.5^{\mathrm{b}}$ & $8^{\mathrm{b}}$ & $3^{\mathrm{a}}$ & 33.9 \\
\hline
\end{tabular}

Bastidas y Triana, (2003) en sus trabajos con plantas acompañantes encontraron información similar a la encontrada en esta investigación, estos autores sostienen que la planta $H$. capitata albergó gran cantidad de Hymenopteros especialmente Telenomus, Trichogramma, Chalcididos y Braconidos con poblaciones entre 20 insectos/planta/día. 
Tabla 9. Identificación de insectos visitantes de Hyptis capitata.

\begin{tabular}{|c|c|c|c|}
\hline Orden & Familia & Género & Observación \\
\hline Coleóptera & $\begin{array}{l}\text { Chrysomelideae } \\
\text { Coccinelideae } \\
\text { Tenebrionideae } \\
\text { Lampyrideae } \\
\text { Carabideae } \\
\text { Scarabeideae } \\
\text { Curculionideae }\end{array}$ & $\begin{array}{l}\text { Hyperaspis } \\
\text { Hipodamia } \\
\text { Coleomegilla }\end{array}$ & $\begin{array}{c}\text { Fitófago } \\
\text { Benéfico } \\
\text { Benéfico } \\
\text { Predador insectos plaga }\end{array}$ \\
\hline & Pentatomideae & $\begin{array}{l}\text { Oebalus } \\
\text { Nezara } \\
\text { Mormidea }\end{array}$ & Plagas en arroz \\
\hline Hemíptera & $\begin{array}{l}\text { Reduviideae } \\
\text { Coreideae } \\
\text { Mirideae } \\
\text { Nabideae } \\
\text { Lygaeideae } \\
\text { Thyreocorideae } \\
\text { Aradideae } \\
\text { Arthideae }\end{array}$ & Leptoglosus & $\begin{array}{l}\text { Enemigo natural plagas } \\
\text { Fitófago Chupador }\end{array}$ \\
\hline Lepidóptera & $\begin{array}{l}\text { Brassolideae } \\
\text { Pompilideae } \\
\text { Antocorideae }\end{array}$ & & $\begin{array}{c}\text { Fitófago } \\
\text { Benéfico } \\
\text { Predador no especifico }\end{array}$ \\
\hline Himenóptera & $\begin{array}{l}\text { Vespideae } \\
\text { Ischneumonideae } \\
\text { Sphecideae } \\
\text { Chalcideae }\end{array}$ & Polidia & Parasitoide \\
\hline Homóptera & $\begin{array}{l}\text { Cicadelideae } \\
\text { Membracideae } \\
\text { Cercopideae }\end{array}$ & Tetragónide & Fitófago \\
\hline Díptera & $\begin{array}{l}\text { Tachinideae } \\
\text { Asílideae }\end{array}$ & Paratheresia & Parasitoide \\
\hline Orthoptera & $\begin{array}{l}\text { Acriideae } \\
\text { Tettigonideae }\end{array}$ & Conocéphalus & Fitófago \\
\hline Odonata & $\begin{array}{l}\text { Libelulideae } \\
\text { Coenagionideae }\end{array}$ & & \\
\hline Neuróptera & $\begin{array}{l}\text { Chrysopideae } \\
\text { Licoccideae }\end{array}$ & & $\begin{array}{c}\text { Predador no especifico } \\
\text { Depredador }\end{array}$ \\
\hline Arácnidos & $\begin{array}{l}\text { Thomicideae } \\
\text { Oxypideae } \\
\text { Araneideae }\end{array}$ & Alpaida & \\
\hline
\end{tabular}


En palma de aceite Calvache, (2001) sostiene que plantas como Hyptis capitata y Crotalaria juncea entre otras, son plantas que proporcionan alimento listo en carbohidratos a los adultos de insectos parasiotides, siendo estas plantas hospederas alternas de insectos benéficos en el agroecosistema de la palma de aceite.

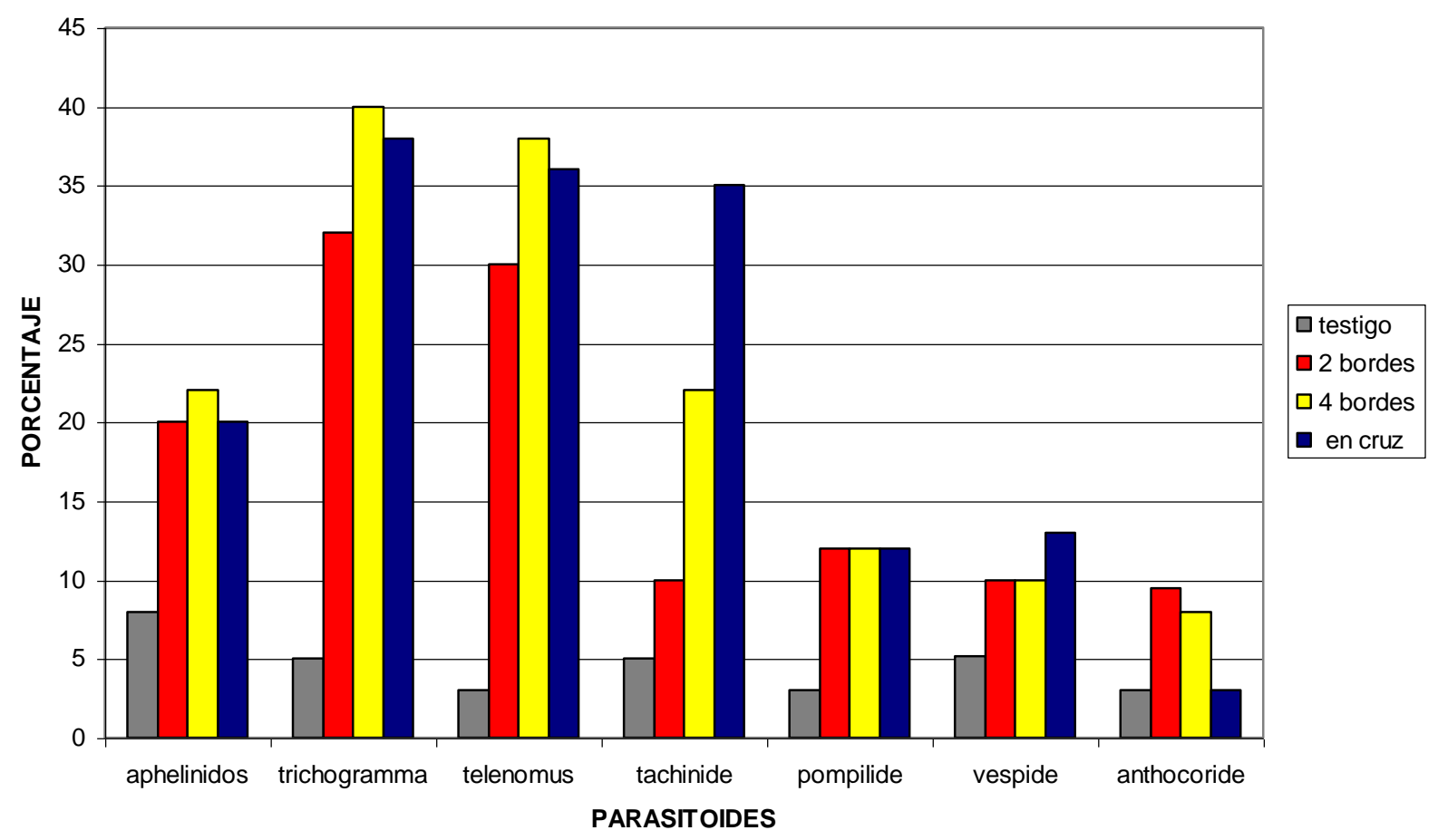

Gráfica 4. Presencia de insectos benéficos en $H$. capitata.

La población de Trichogramma spp, Telenomus y moscas de la Familia Tachinidae fue mayor en la distribución en los bordes y en cruz (Tabla 8), esto se debe a la cantidad de flores que hay cuando se siembran mayor cantidad de plantas, también se encontró que la cantidad de azucares que posee esta planta influye y sobre todo cuando la densidad de plantas se aumenta. Las avispas Trichogramma y Telenomus fueron las más capturadas en este estudio. Bastidas y Triana, (2003) seleccionaron a $H$. capitata y Crotalaria juncea como plantas acompañantes promisorias para desarrollar proyectos de investigación en esta área. 
En huevos de pentastómidos el mayor parasitismo se encontró en disposición en cruz con $9.5 \%$ presentando diferencias estadísticas significativas con los otros tratamientos utilizados (Tabla 10), resultados similares son reportados por Bastidas y Triana, (2003) quienes afirman que cuando se utilizo $H$. capitata como cultivo acompañante el porcentaje de parasitismo fue mayor en huevos de pentastómidos, huevos y larvas de Spodoptera y huevos de Mocis comparado con otro cultivo acompañante como Crotalaria, y el sitio sin cultivo acompañante.

Tabla 10. Porcentaje de parasitismo en H. capitata.

\begin{tabular}{lccccc}
\hline \multicolumn{1}{c}{ Tratamiento } & Testigo & 2 Bordes & 4 Bordes & En Cruz & CV \\
\hline Huevos & $1^{\mathrm{a}}$ & $8^{\mathrm{b}}$ & $12^{\mathrm{c}}$ & $9.5^{\mathrm{b}}$ & 18.5 \\
pentastómidos & $3^{\mathrm{a}}$ & $18^{\mathrm{b}}$ & $16^{\mathrm{b}}$ & $18^{\mathrm{b}}$ & 12.8 \\
Huevos Diatrea & $0^{\mathrm{a}}$ & $10^{\mathrm{b}}$ & $10^{\mathrm{b}}$ & $10^{\mathrm{b}}$ & 13.3 \\
Huevos Salbia & $3^{\mathrm{a}}$ & $12^{\mathrm{b}}$ & $20^{\mathrm{d}}$ & $18^{\mathrm{c}}$ & 8.5 \\
Larvas Salbia & $5^{\mathrm{a}}$ & $8^{\mathrm{b}}$ & $14^{\mathrm{c}}$ & $16^{\mathrm{c}}$ & 17 \\
Larvas & & & & & \\
Spodopthera & & & &
\end{tabular}

El parasitismo en huevos de Diatraea fue similar estadísticamente en todas las disposiciones utilizadas, lo que indica que con baja densidad de plantas solo en un borde la eficiencia y la efectividad del cobertor son apropiadas. Para el caso de huevos de Salbia parasitadas por Trichogramma presentaron las mismas características que los huevos de Diatraea. Lo que indica que este cobertor es eficiente como albergue de Trichogramma el principal parasitoide de huevos de Diatraea. En el caso de larvas de Lepidópteros parasitados por Tachinidos y Braconidos presentaron mayor incidencia con los cuatro bordes y la disposición en cruz, debido a que estos parasitoides requieren mayor población de plantas refugio para que su acción sea eficiente (Tabla 10) (Gráfica 5).

\section{Ensayo 6. Efecto de herbicidas sobre $\boldsymbol{H}$. capitata}

\section{Pruebas de pre-emergencia}

Dentro del análisis estadístico se logra establecer dos rangos de control diferenciados, como lo es la altísima eficiencia del oxadiazon con un $99.66 \%$ y el 
butaclor (94.43\%) el siguiente rango lo establece el oxyfluorfen con un control del $77.76 \%$, pero no muy distante el pretilaclor con un control del $73.63 \%$ (Tabla 11).

Tabla 11. Análisis estadístico prueba de herbicidas pre-emergentes.

\begin{tabular}{lcc}
\hline \multicolumn{1}{r}{ Producto } & $\begin{array}{c}\text { Dosis } \\
\text { (L/ha) }\end{array}$ & $\begin{array}{c}\text { Eficiencia del producto } \\
(\%)\end{array}$ \\
\hline Pretilaclor & 2,5 & $73.65^{\mathrm{b}}$ \\
Butaclor & 4,0 & $94.43^{\mathrm{a}}$ \\
Oxadiazon & 2,0 & $99.66^{\mathrm{a}}$ \\
Oxyfluorfen & 1,2 & $77.76^{\mathrm{b}}$ \\
Testigo & 0 & $0^{\mathrm{c}}$ \\
\hline
\end{tabular}

C.V. $=7.5 \%{ }^{* *}$ Diferencia altamente significativa $1 \%$.

NOTA: Diferencia con igual letra indica no poseer diferencia estadísticamente significativa.

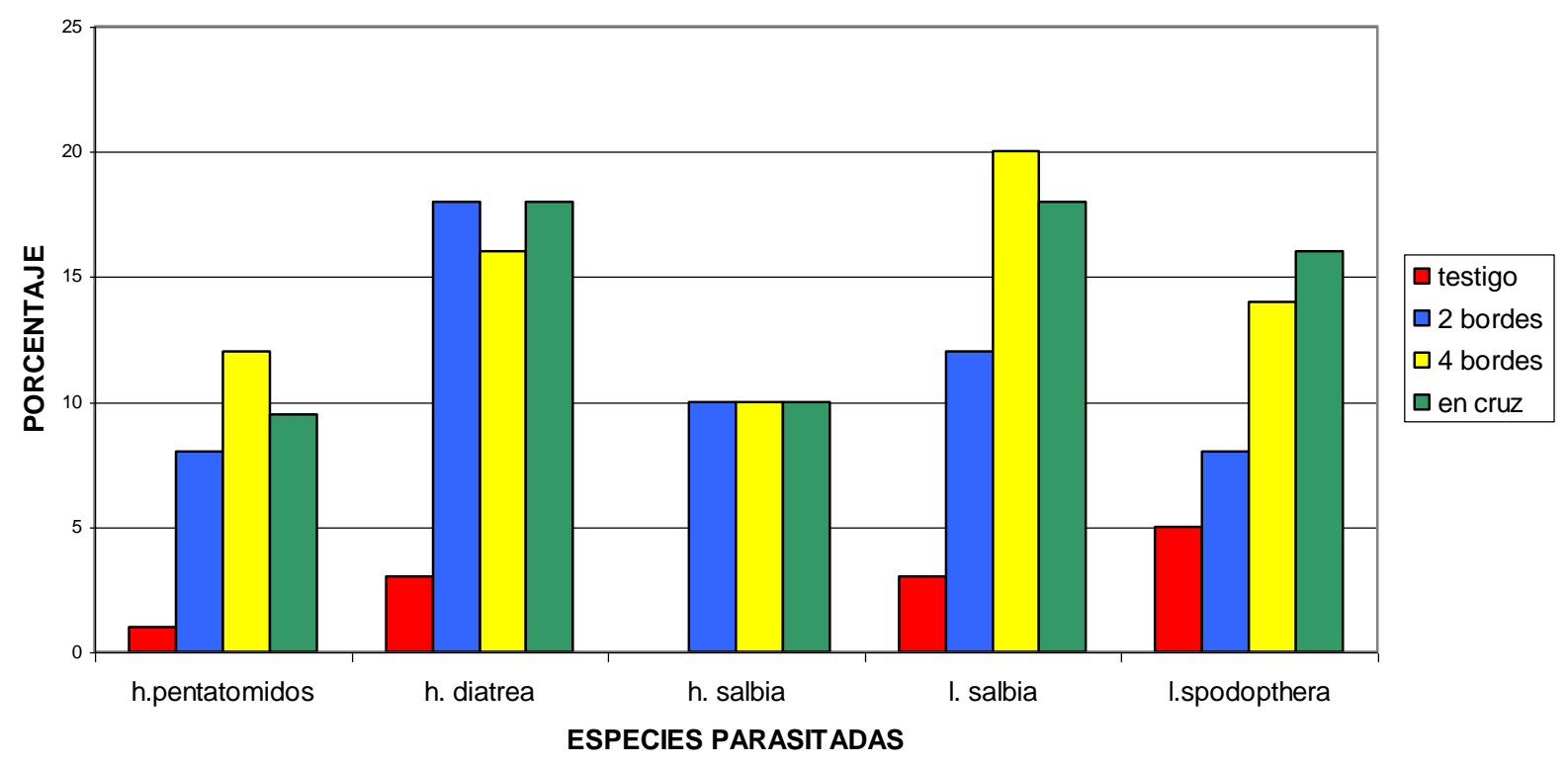

Gráfica 5. Porcentaje de parasitismo influenciado por la presencia de Hyptis

\section{Prueba de post-emergentes}

Los herbicidas de mayor desempeño son aquellos a base de metsulfuron $94.25 \%$, Byspiribac sodio $93.5 \%$ y azimsulfuron + metsulfuron 95\%, los cuales no presentan diferencia estadística entre ellos. El herbicida que presentó una diferencia estadísticamente baja corresponde al propanil en dosis de 4 litros 
(75.5\%); aunque propanil 5 litros y 2,4 D amina demostraron un comportamiento muy similar (Tabla 12).

Tabla 12. Análisis de eficiencia herbicidas post-emergentes.

\begin{tabular}{lcc}
\hline \multicolumn{1}{c}{ Ingrediente activo } & $\begin{array}{c}\text { Dosis } \\
\text { comercial/ha }\end{array}$ & $\begin{array}{c}\text { Eficiencia del } \\
\text { producto (\%) }\end{array}$ \\
\hline 2,4D & $0.4 \mathrm{~L}$ & $81.50^{\mathrm{ab}}$ \\
2.4 D + Piclosan & $0.4 \mathrm{~L}$ & $85.50^{\mathrm{b}}$ \\
Azimsulfuron + Metsulfuronmetil & $0.01 \mathrm{Kg}$ & $95.00^{\mathrm{c}}$ \\
Metsulfuronmetil & $0.015 \mathrm{Kg}$ & $94.25^{\mathrm{c}}$ \\
Bentazona & $2.5 \mathrm{~L}$ & $85.62^{\mathrm{b}}$ \\
Loxinil octanoato & $0.3 \mathrm{~L}$ & $85.00^{\mathrm{b}}$ \\
Propanil + Trycorpir & $4.0 \mathrm{~L}$ & $85.50^{\mathrm{b}}$ \\
Propanil & $4.0 \mathrm{~L}$ & $75.50^{\mathrm{a}}$ \\
Propanil & $5.0 \mathrm{~L}$ & $79.87^{\mathrm{ab}}$ \\
Byspiribac Sodio & $0.5 \mathrm{~L}$ & $93.50^{\mathrm{c}}$ \\
Testigo & & $0^{\mathrm{d}}$ \\
\hline
\end{tabular}

C.V. $=5.2 \%{ }^{* *}$ Diferencia altamente significativa $1 \%$.

NOTA: Diferencia con igual letra indica no poseer diferencia estadísticamente significativa.

\section{CONCLUSIONES}

- El mejor método de escarificación para disponer de plántulas en investigación o como corredores biológicos es disponer la semilla en una estufa a $65^{\circ} \mathrm{C}$ durante 72 horas.

- La germinación de la especie es mayor en la superficie ó hasta los dos centímetros de profundidad.

- La capacidad de persistencia de esta especie no radica en su latencia, ó en su porcentaje de germinación ya que son muy bajas; sino en la capacidad de producir una alta cantidad de semillas (más de 3000 semillas por planta).

- Dentro de la alta cantidad de especies de insectos que llegan las plantas de Hyptis capitata, la que se presenta en mayor cantidad son las de los ordenes Hemíptera, Coleóptera, Himenóptera y de la clase Arácnida. Los cuales poseen un alto número de benéficos entre sus representantes como Reduviideae, Calcideae, Pompilideae, entre otras. 
- Si se emplean herramientas de tipo químico para el control de Hyptis capitata Jaqc, dentro de los herbicidas pre-emergentes el de mejor desempeño es el oxadiazon y butaclor. Los mejores herbicidas postemergentes para el control de falso cordón de fraile es son Azimsulfuron + Metsulfuronmetil, Byspiribae Sodio y Metsulfuronmetil.

- La mayor población de insectos benéficos se presenta en horas de la mañana y la más baja se encuentra en horas del medio día; con excepción de los insectos del orden Hymenóptera.

\section{RECOMENDACIONES}

- Según el estudio realizado es recomendable la utilización de Hyptis capitata Jacq como especie atrayente de insectos benéficos en el cultivo de arroz, debido a la relación favorable entre enemigos naturales e insectos plagas atraídos por esta especie.

- Para el cultivo del arroz, esta especie se podría emplear en los bordes del lote para la atracción de artrópodos benéficos.

\section{REFERENCIAS BIBLIOGRÁFICAS}

1. Association of Official Analytical Chemists (A.O.A.C.). Official Methods of Analysis. 18 ${ }^{\text {th }}$ Ed., Washington, D. C. 2006.

2. Association of Official Analytical Chemists (A.O.A.C.) Official Methods of Analytical Association of Official Analytical Chemical. Washington, D.C. 1980.

3. Bastidas H. Generalidades de malezas en los Llanos orientales de Colombia. Pendiente de publicación. Cartilla de capacitación. Villavicencio. Fedearroz Diciembre. 35 p. 2001.

4. Bastidas H., Triana, M. Utilización de plantas acompañantes o refugio de enemigos naturales en el cultivo del arroz. Boletín informativo del FLAR. Colombia. 9 (2): 24-28. 2003.

5. Bastidas H., Ordoñez R. Se incrementan malezas en los Llanos Orientales. Revista Arroz. 50 (434): 4-8. 2001.

6. Calvache H., Franco P., Aldana J., Aldana R. Plagas de la palma de aceite en Colombia. CENIPALMA, Santafé de Bogotá. p. 81-85. 2000.

7. Cárdenas, J., Reyes, C., Doll, J., Pardo, F. Malezas tropicales, ICA. 1: 178179. 1972. 
8. Cronquist A. Introducción a la botánica. Compañía editorial continental S.A. de C.V. p 126. 1992.

9. Ghia S. Situación de la investigación de plantas medicinales en la Amazonía; panorámica de la investigación en los países. 1993. Disponible En: www.amazonas.rds.org.co.

10. Gómez J. Control químico de malezas. Editorial Trillas, p 7-8. 1993.

11. Gómez A., Rivera H., Descripción de malezas en plantaciones de café. CENICAFÉ, p 268-300. 1987.

12. Izco J., Barreno E., Costa M., Devesa J., Fernández F., Gallardo T., Llimona, X., Salvo, E., Talavera, S., Valdés B. Botánica. McGraw Hill, p 519- 524. 2000.

13. Marzocca A. Taxonomía Vegetal, Instituto Interamericano de Cooperación para la agricultura. Costa Rica. p 40-41. 1985.

14. Mexzon R., Chinchilla C. Especies vegetales atrayentes de la entomofauna benéfica en plantaciones de palma aceitera (Elaeais guianeensis) en Costa rica. 1999. Disponible En: www.asd-cr.com

15. Salamanca G., Salamanca P., Pérez F., Zapata M., Rivera F., Osorio P. Flora apical indicadora del departamento del Tolima. 2001

16. Takhtajan. Columbia University Press Newyork. p 423-467. 1997. 